\title{
Perspective on the Optimal Diagnostic Approach for Dysphagia Considering Wearing-off Phenomenon in a Patient with Parkinson's Disease: A Case Report
}

Kyoung Tae Kim ( $\nabla$ zealot42@dsmc.or.kr)

Keimyung University Dongsan Hospital, Keimyung University School of Medicine

\section{Case Report}

Keywords: wearing-off phenomenon, Parkinson's disease, dysphagia, videofluroscopic swallowing study

Posted Date: February 21st, 2022

DOI: https://doi.org/10.21203/rs.3.rs-1374826/v1

License: (c) (i) This work is licensed under a Creative Commons Attribution 4.0 International License.

Read Full License 


\section{Abstract}

\section{Background}

Dysphagia is a major disability in patients with Parkinson's disease. Unlike its motor symptoms, dysphagia is relatively unresponsive to dopaminergic drugs. However occasionally, swallowing difficulty is significantly affected by the wearing off phenomenon. In such cases, it is difficult to accurately assess the severity of dysphagia if evaluation of swallowing is performed without considering the on-period based on dopaminergic medication.

\section{Case presentation}

Here, we report a case of dysphagia in a patient with Parkinson's disease severely affected by the wearing-off phenomenon. The patient presented severe oral phase delay with choking symptoms during fluid intake. Massive aspiration was observed on routine scheduled videofluroscopic swallowing study, and an alternative feeding method and maximum diet restriction were prescribed. However, when swallowing evaluation was performed according to the on-period, almost no abnormal function was observed, and after the on/off period-tailored food intake prescription, sufficient nutrition was possible without aspiration.

\section{Conclusion}

In swallowing evaluation for dysphagia in patient with Parkinson's disease, it is essential to consider the wearing-off phenomenon. Patient-specific swallowing evaluation and diet prescription is needed to establish optimal therapeutic strategies.

\section{Background}

Dysphagia is a major disability in patients with Parkinson's disease. It can result in malnutrition, aspiration pneumonia, decreased quality of life, and increased overall mortality (1). Swallowing difficulty is detected in a relatively later phase after the diagnosis of Parkinson's disease compared to motor symptoms and is often underestimated due to certain silent aspirations. Here, we report dysphagia case in patient with Parkinson's disease severely affected by wearing-off phenomenon.

\section{Case Presentation}

A 59-year-old woman with Parkinson's disease visited our clinic for evaluation of swallowing dysfunction. She presented with severe limb rigidity, postural instability with Hoehn and Yahr stage 3, dysarthria, pathological crying, and irritability. She was taking levodopa with benserazide (Madopar) for a total of 5 times a day $(187.5 \mathrm{mg}, 218.75 \mathrm{mg}, 218.75 \mathrm{mg}, 218.75 \mathrm{mg}, 187.5 \mathrm{mg}$ at $10 \mathrm{AM}, 11: 30 \mathrm{AM}, 2: 00 \mathrm{PM}, 4: 00$ PM, and 6:00 PM, respectively). She experienced a severe wearing-off phenomenon of levodopa response. She could walk with assistance and was able to use her hands for daily activities during the on- 
period, from around 11:00 AM to 7 PM, but was totally dependent on a wheelchair with pathological crying, hallucinations, and delirium during the off-period. The swallowing difficulty developed several months ago, with frequent aspiration of liquids. We performed a videofluoroscopic swallow study (VFSS) at approximately 9:00 AM, which is the regular evaluation schedule in our clinic. With $5 \mathrm{ml}$ of nectar-thick liquid, the esophagogram showed tongue disorganization, delayed oral transit time, and lack of laryngeal elevation with impairment of laryngeal vestibule closure. Aspiration was observed during swallowing, using the penetration-aspiration scale (PAS) 8 (Figure. 1A). A similar abnormality was observed with $3 \mathrm{ml}$ of a thin liquid (PAS 8) (Figure. 1B). Semi-solid testing using rice porridge showed markedly reduced mastication and persistent retention of food bolus in the oral cavity without any propulsion to the pharyngeal phase. Based on the results of VFSS, we recommended an alternative method of feeding considering the lack of sufficient nutrition and hydration with maximum diet restriction. However, the patient and her caregiver refused our diet prescription because of previous oral food intake.

After a more detailed history-taking, we noted that the patient's swallowing function was severely affected by the wearing-off cycle. She was on a regular diet without any restriction during the on-period between 12:00 AM to 6:00 PM, but had frequent choking and deglutition during the off-period in the morning and late afternoon. Considering the fluctuation in swallowing difficulty, we performed the VFSS again at approximately 2:00 PM. VFSS showed a significant improvement in swallowing function compared to that in the previous study. There was no aspiration, and the laryngeal elevation with LVC was nearly intact. In particular, mastication, lip closure, and tongue disorganization were improved noticeably, reducing the oral transit time (Figure. 2A, B). She could swallow semi-solid and solid diets without any aspiration or time delay (Figure. 2C, D). Regarding the VFSS, we decided to try oral intake with food modification using a soft-minced diet and fluid with a thickener only during the on-period of the day. Most of her swallowing difficulties were alleviated, and sufficient nutritional support was also possible.

\section{Discussion And Conclusions}

Dysphagia is one of the most common complications of nonmotor symptoms in patients with Parkinson's disease. The prevalence of dysphagia is reported to vary from $18-100 \%$, and it decreases quality of life while increasing over-all mortality (2). Although typical motor symptoms, including bradykinesia and rigidity, can cause dysphagia, dopaminergic medication reportedly has little effect on dysphagia in PD $(3,4)$. The involvement of extra-striatal and non-dopaminergic systems associated with the basal ganglia may play a crucial role in swallowing function (5). A previous meta-analysis revealed an absence of correlation between levodopa intake and improvement in swallowing (1). Although these characteristics of dysphagia are common in patients with PD, some patients have a significant change in swallowing function depending on levodopa administration. VFSS has shown a remarkable improvement in the swallowing function during the on-period. In such cases, routine swallowing evaluation without considering the wearing-off effect does not accurately evaluate the comprehensive swallowing function, leading to overestimation of the severity of dysphagia. Moreover, enteral feeding may result in the loss of the patient's enjoyment of oral intake and a poor quality of life. In conclusion, detailed history-taking 
regarding the on-off period of dysphagia according to levodopa intake is needed. Furthermore, a patienttailored swallowing evaluation should be performed to establish optimal treatment strategies.

\section{Abbreviations}

VFSS : videofluoroscopic swallow study

\section{Declarations}

\section{Ethics approval and consent to participate}

This study was approved by the Institutional Review Board of Keimyung University Dongsan Hospital (IRB No: 2021-12-022).

\section{Availability of data and materials}

Data and materials related to the current study are available from our corresponding author upon reasonable request.

\section{Competing interests}

No potential conflict of interest relevant to this article was reported.

\section{Funding}

This research received no specific grant from any funding agency in the public, commercial, or not-forprofit sectors.

\section{Acknowledgements}

We would like to thank the patient and doctors who participated in this study for their cooperation

\section{References}

1. Takizawa C, Gemmell E, Kenworthy J, Speyer R. A systematic review of the prevalence of oropharyngeal dysphagia in stroke, Parkinson's disease, Alzheimer's disease, head Injury, and pneumonia. Dysphagia. 2016;31(3):434-41. http://doi.org/10.1007/s00455-016-9695-9.

2. Miller N, Allcock L, Hildreth AJ, Jones D, Noble E, Burn DJ. Swallowing problems in Parkinson disease: frequency and clinical correlates. Journal of Neurology, Neurosurgery \&amp; Psychiatry. 2009;80(9):1047-9. http://doi.org/10.1136/jnnp.2008.157701.

3. Hunter PC, Crameri J, Austin S, Woodward MC, Hughes AJ. Response of parkinsonian swallowing dysfunction to dopaminergic stimulation. J Neurol Neurosurg Psychiatry. 1997;63(5):579-83. http://doi.org/10.1136/jnnp.63.5.579. 
4. Menezes $C$, Melo A. Does levodopa improve swallowing dysfunction in Parkinson's disease patients? J Clin Pharm Ther. 2009;34(6):673-6. http://doi.org/10.1111/j.1365-2710.2009.01031.x.

5. Storch A, Schneider CB, Wolz M, Stürwald Y, Nebe A, Odin P, et al. Nonmotor fluctuations in Parkinson disease: severity and correlation with motor complications. Neurology. 2013;80(9):800-9. http://doi.org/10.1212/WNL.0b013e318285c0ed.

\section{Figures}
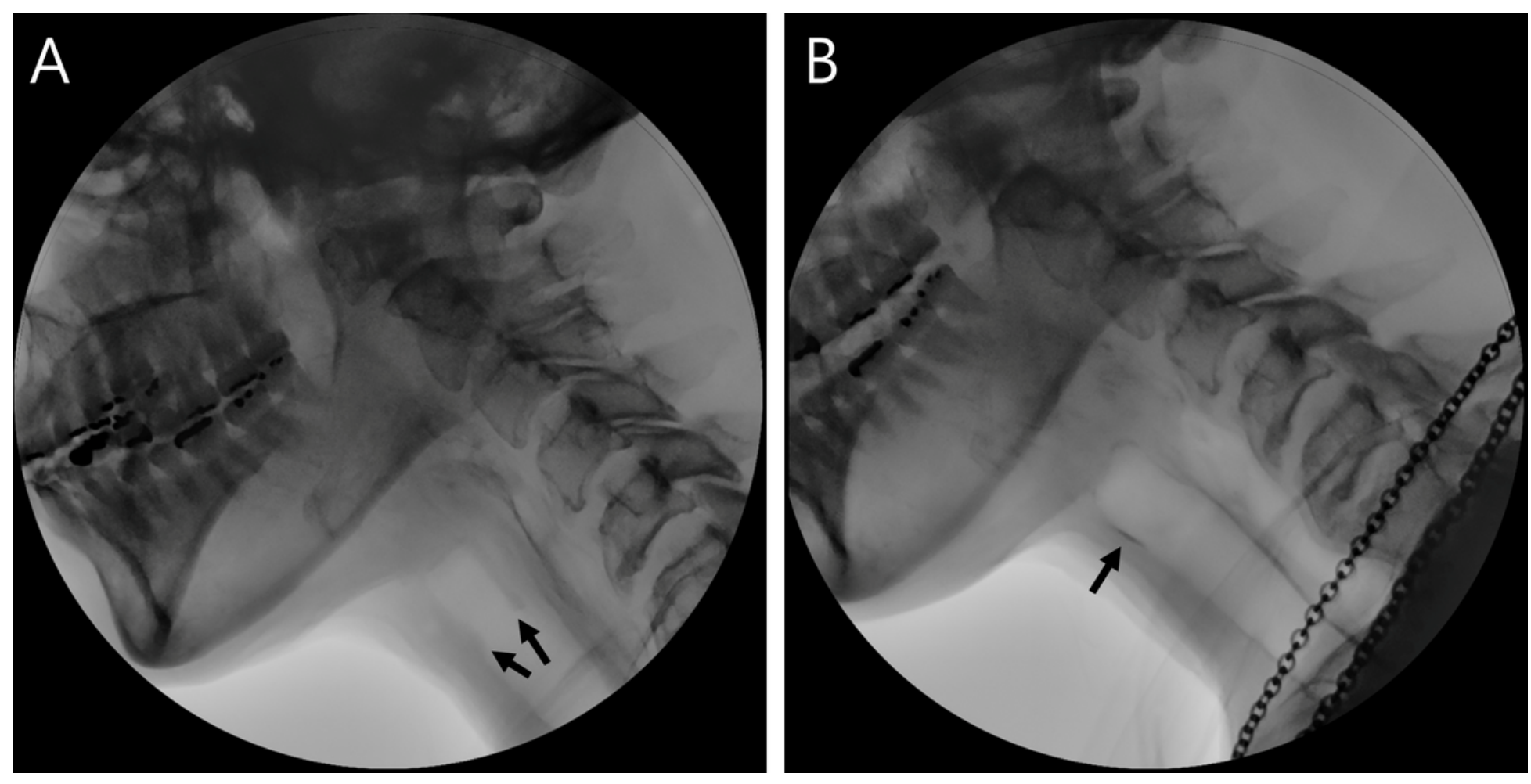

Figure 1

Videofluoroscopic swallow study during the off-period in a patient with Parkinson's disease showed massive aspiration. (a) Nectar-thick liquid and (b) $3 \mathrm{ml}$ of a thin liquid. Arrow: aspiration 

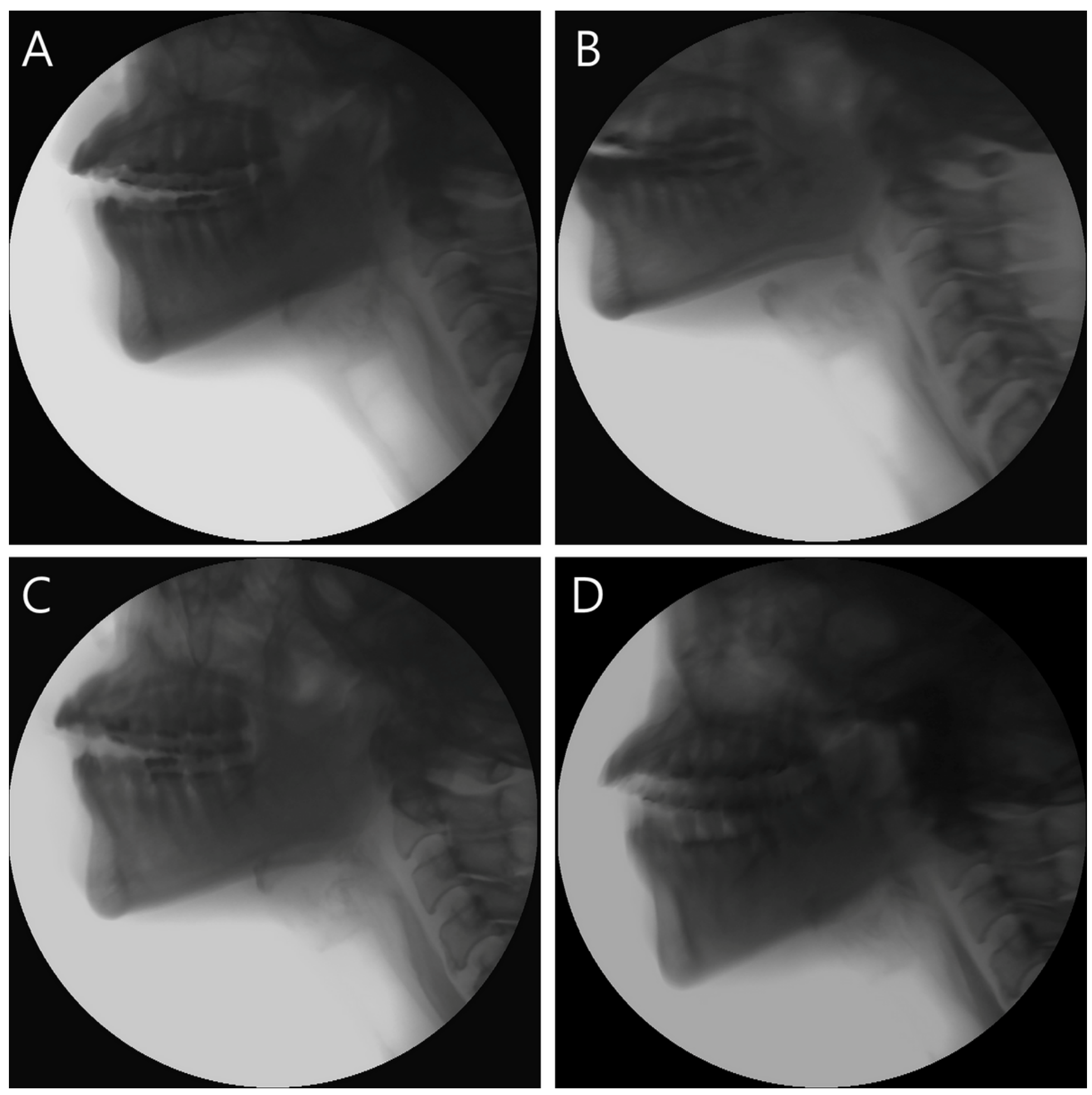

Figure 2

Videofluoroscopic swallow study during the on-period in a patient with Parkinson's disease without any aspiration. (a) Nectar-thick liquid; (b) $5 \mathrm{ml}$ of a thin liquid; (c) semi-solid; and (d) solid. 